Article

\title{
Silica-Supported Polyphosphoric Acid in the Synthesis of 4-Substituted Tetrahydroisoquinoline Derivatives
}

\author{
Stanimir Manolov, Stoyanka Nikolova and Iliyan Ivanov * \\ Department of Organic Chemistry, University of Plovdiv, 24 Tzar Assen str., 4000 Plovdiv, Bulgaria \\ * Author to whom correspondence should be addressed; E-Mail: iiiliyan@abv.bg or \\ ivanov@uni-plovdiv.bg; Tel.: +359-32-261-349; Fax: +359-32-261-403.
}

Received: 19 December 2012; in revised form: 21 January 2013 / Accepted: 22 January 2013 / Published: 1 February 2013

\begin{abstract}
We report herein an application of an $\alpha$-amidoalkylation reaction, as an alternative efficient synthesis of 4-aryl- and 4-methyl-1,2,3,4-tetrahydroisoquinoline derivatives. The amides required for this purpose would result from reaction of aminoacetaldehyde dimethylacetal with different substituted benzenes in polyphosphoric acid, followed by acylation of the obtained amines with different acid chlorides or sulfochlorides. We compared the cyclisation step using conventional (milieu of acetictrifluoracetic acid $=4: 1)$ and solid supported reagents $\left(\mathrm{SiO}_{2} / \mathrm{PPA}\right)$, as recovered, regenerated and reused without loss of its activity catalyst. We found that in comparison to conventional methods, the yields of the reaction are greater and the reaction time is shorter.
\end{abstract}

Keywords: 4-substituted tetrahydroisoquinoline; cherylline analogues; $\alpha$-amidoalkylation reaction; silica-supported polyphosphoric acid $\left(\mathrm{SiO}_{2}-\mathrm{PPA}\right)$

\section{Introduction}

Very rare natural 4-aryl-1,2,3,4-tetrahydroisoquinoline alkaloids have been isolated from Crinum powellii var. alba and other Crinum species [1]. Due to the uniqueness of the structure and potential medicinal properties of the 4-arylisoquinoline derivatives [2-5], many synthetic routes for these compounds [6-15] and especially cherylline have been reported. The Amaryllidaceae [6-16] have been one of the most studied families of plants because of their alkaloids composition. In 1970, Brossi et al. [1] isolated from Crinum powelli an alkaloid with a 4-aryl-1,2,3,4-tetrahydroisoquinoline structure named cherylline. Some of them are valuable medicines with centrally stimulating, 
thymoleptic, and antiarrhythmic actions [2,17]. Others are calcium antagonistic [18], and exhibit broad spectrum of activity such as antibacterial [19], antiplasmodial [3,4,16,20,21], estrogen agonist/antagonist activity [22], and serotin (5-HT) re-uptake [23].

The importance of these heterocyclic compounds has generated a considerable number of synthetic approaches. Hence, the formation of the nitrogen-containing ring of 4-substituted 1,2,3,4tetrahydroisoquinolines has been achieved with several reactions such as Bischler-Napieralski [24-26], intramolecular Horner [27], Friedel-Crafts [21,27-32] Pictet-Spengler [33], and oxazoline-driven chemistry [34], among others [25,35-42]. Most of the reported methods for the synthesis of these compounds are either multistep or low yielding.

In our previous investigations we report application of polyphosphoric acid (PPA) as a cyclisation agent for the construction of tetrahydroisoquinoline ring systems. Polyphosphoric acid is a strong mineral acid which has powerful dehydrating properties and is widely used for intramolecular and intermolecular acylations, heterocyclic synthesis, and acid-catalyzed reactions. For instance, in recent reports the cyclization reactions of $N, N^{\prime}$-bis(oxotrifluoroalkenyl)-1,3-phenylenediamines in PPA medium [43], the acylation of benzene and its derivatives with 2-, 3-, 4-aminobenzoic and 4-aminophenylacetic acid in PPA to aminobenzophenones [44], and the reaction of tryptamine with carboxylic acid in PPA to afford 1-substitued-3,4-dihydro-9H- $\beta$-carboline derivatives [45], etc, were described. However, the use of PPA has several drawbacks: since 10- to 50-fold excess is generally employed, it is difficult to pour and stir at room temperature, and it is necessary to carefully neutralize the reaction mixtures before the product extraction.

Recently, PPA/SiO 2 has been used as an efficient heterogeneous catalyst for many organic transformations. $\mathrm{PPA} / \mathrm{SiO}_{2}$ has some advantages including its low cost, ease of preparation, and ease of handling. In addition, the catalyst can be easily separated from the reaction mixtures by simple filtration and is reusable. Previously [46], the conversion of carbonyl compounds into oxathioacetals or dithioacetals using PPA/SiO 2 and a convenient method for the synthesis of isoxazole derivatives using $\mathrm{PPA} / \mathrm{SiO}_{2}$ as a reusable catalyst have been reported [47]. In the last several years the development of non-toxic, low cost, eco-friendly, recycable catalyst systems which give high productivity under mild reaction conditions has received much attention in organic synthesis [48]. Solid supported catalysts [49,50] have gained much prominence due to their inherent economic and environmental benefits, ease of handling, easy catalyst separation and regeneration, thermal stability and long catalytic life [51]. Since the activity and selectivity of a reagent dispersed on the surface of the support is improved as the effective surface area of reagent can be increased manifold, they are expected to perform better than the individual reagents [52]. Low toxicity, moisture, air tolerance and low price are other common features that make the use of solid supported reagents attractive alternative to the conventional catalysts.

\section{Results and Discussion}

Our retrosynthetic analysis of 4-substituted 1,2,3,4-tetrahydroisoquinolines is depicted in Scheme 1. We anticipated that 5 could be constructed from amides $\mathbf{4}$ via an $\alpha$-amidoalkylation reaction. The required amides $\mathbf{4}$ can be prepared from the amines $\mathbf{3}$ via acylation with different acid chlorides. The amines 3 would arise from reaction [53] of aminoacetaldehyde dimethylacetal (1) with benzene or substituted benzenes 2 (Scheme 2). 
Scheme 1. Retrosynthetic scheme for the synthesis of 4-substituted tetrahydroisoquinolines.<smiles>[R]c1ccc(C2CN([R2])Cc3cc([R])c([R])cc32)cc1[R]</smiles>

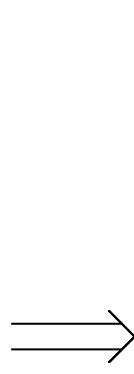

$\mathrm{R}_{1}=\mathrm{H}, \mathrm{OMe}$ $\mathrm{R}_{2}=\mathrm{COCH}_{3}, \mathrm{SO}_{2} \mathrm{CH}_{3}, \mathrm{COPhPh}$<smiles>[R2]NCC(c1ccc([R])c([R])c1)c1ccc([R])c([R])c1</smiles>

4<smiles>[R]c1ccc(C(CN)c2ccc([R])c([R])c2)cc1[R]</smiles>

Scheme 2. Synthesis of amines 3.<smiles>COC(CN)OC</smiles><smiles>[R]c1ccccc1C(C)C</smiles><smiles>[R]c1ccc(C(CN)c2ccc([R])c([R])c2)cc1[R]</smiles>

For the synthesis of the required starting materials Klumpp's [53] protocol using amino acetals and benzene in $\mathrm{TfOH}$ to give the corresponding products with good yields can be used. We carried out the same reaction in polyphosphoric acid instead of $\mathrm{TfOH}$. We found that aminoacetaldehyde dimethylacetal reacts with excess of 1,2-dimethoxybenzene (veratrol) in PPA at rt for 5 to 10 min to give a good $(82 \%)$ yield of the corresponding product 3 . The same product with unsubstituted benzene was obtained after $30 \mathrm{~min}$ at $80{ }^{\circ} \mathrm{C}$ with $42 \%$ yield (Scheme 2 , Table 1).

Table 1. Synthesis of amines 3.

\begin{tabular}{|c|c|c|c|c|}
\hline 3 & \multicolumn{2}{|c|}{$\mathbf{R}_{1}$} & Yield, \% & M.p., ${ }^{\circ} \mathbf{C}$ \\
\hline $\mathbf{a}$ & $\mathrm{H}$ & $\mathrm{H}$ & 42 & 47-49 \\
\hline b & $\mathrm{OCH}_{3}$ & $\mathrm{OCH}_{3}$ & 82 & oil \\
\hline
\end{tabular}

The amides 4 required for the next step of our synthesis, were prepared by acylation of the amines 3 with different acid chlorides or sulfochlorides. The next step was cyclization of the newly synthesized amides (Scheme 3, Table 2). 
Scheme 3. Synthesis of 4-aryl tetrahydroisoquinolines.

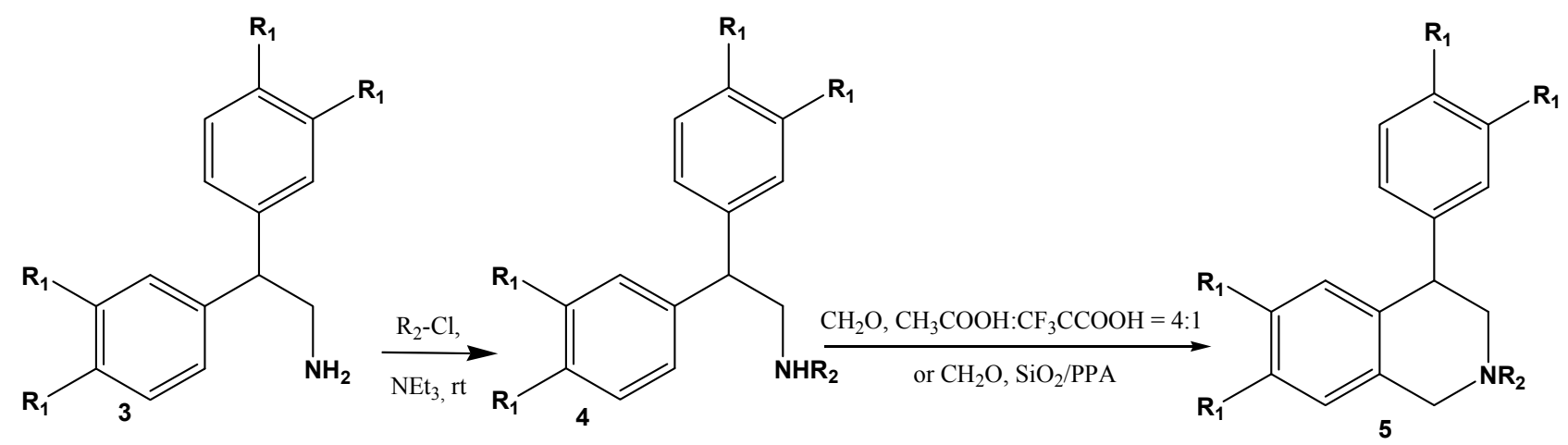

Table 2. Synthesis of amides 4.

\begin{tabular}{cccccc}
\hline $\mathbf{4}$ & $\mathbf{R}$ & $\mathbf{R}_{\mathbf{1}}$ & $\mathbf{R}_{\mathbf{2}}$ & Yield, \% & M.p., ${ }^{\circ} \mathbf{C}$ \\
\hline $\mathbf{a}$ & $\mathrm{H}$ & $\mathrm{H}$ & $\mathrm{COCH}_{3}$ & 80 & $85-86$ \\
$\mathbf{b}$ & $\mathrm{H}$ & $\mathrm{H}$ & $\mathrm{SO}_{2} \mathrm{CH}_{3}$ & 98 & $138-139$ \\
$\mathbf{c}$ & $\mathrm{H}$ & $\mathrm{H}$ & $\mathrm{COPhPh}$ & 94 & $192-193$ \\
$\mathbf{d}$ & $\mathrm{OCH}_{3}$ & $\mathrm{OCH}_{3}$ & $\mathrm{COCH}_{3}$ & 91 & $126-128$ \\
e & $\mathrm{OCH}_{3}$ & $\mathrm{OCH}_{3}$ & $\mathrm{SO}_{2} \mathrm{CH}_{3}$ & 90 & $49-52$ \\
$\mathbf{f}$ & $\mathrm{OCH}_{3}$ & $\mathrm{OCH}_{3}$ & $\mathrm{COPhPh}$ & 97 & $200-202$ \\
\hline
\end{tabular}

We compared the cyclisation step using either mixture of acetic-trifluoracetic acid $=4: 1$ or silica-supported PPA ( $\left.\mathrm{SiO}_{2}-\mathrm{PPA}\right)$. We found that with $\mathrm{SiO}_{2}-\mathrm{PPA}$ the reaction yields are greater and the reaction times are shorter (Table 3 ). The shortest time and best yield were achieved at $80{ }^{\circ} \mathrm{C}$, using $0.06 \mathrm{~g}$ of catalyst and $1 \mathrm{~h}$ reflux. As described earlier [54] the catalyst was recovered quantitatively and used in the reaction three times. The recovered catalyst did not show any significant loss of activity.

Table 3. Comparative yields of the compounds 5 synthesized through two conventional methods.

\begin{tabular}{|c|c|c|c|c|c|}
\hline 5 & $\mathbf{R}$ & $\mathbf{R}_{1}$ & $\mathbf{R}_{\mathbf{2}}$ & $\begin{array}{c}\text { Yield, } \% \\
\left(\mathrm{CH}_{3} \mathrm{COOH}: \mathrm{CF}_{3} \mathrm{COOH}=4: 1\right)\end{array}$ & $\begin{array}{c}\text { Yield, \% } \\
\left(\mathrm{SiO}_{2} / \mathrm{PPA}\right)\end{array}$ \\
\hline $\mathbf{a}$ & $\mathrm{H}$ & $\mathrm{H}$ & $\mathrm{COCH}_{3}$ & 90 & 94 \\
\hline b & $\mathrm{H}$ & $\mathrm{H}$ & $\mathrm{SO}_{2} \mathrm{CH}_{3}$ & 80 & 91 \\
\hline c & $\mathrm{H}$ & $\mathrm{H}$ & $\mathrm{COPhPh}$ & 84 & 96 \\
\hline d & $\mathrm{OCH}_{3}$ & $\mathrm{OCH}_{3}$ & $\mathrm{COCH}_{3}$ & 85 & 96 \\
\hline $\mathbf{e}$ & $\mathrm{OCH}_{3}$ & $\mathrm{OCH}_{3}$ & $\mathrm{SO}_{2} \mathrm{CH}_{3}$ & 87 & 97 \\
\hline f & $\mathrm{OCH}_{3}$ & $\mathrm{OCH}_{3}$ & $\mathrm{COPhPh}$ & 87 & 98 \\
\hline
\end{tabular}

The same protocol was applied for the synthesis of 4-methyl-1,2,3,4-tetrahydroisoquinoline derivatives which are interesting as potential anaesthetics and antispasmodics [55] (Scheme 4, Table 4). 
Scheme 4. Synthesis of 4-methyl tetrahydroisoquinolines.

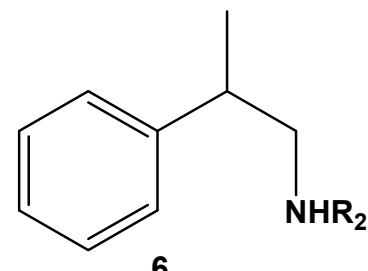

6

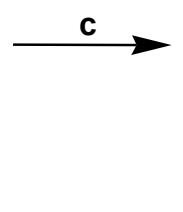

Reagents and Conditions:

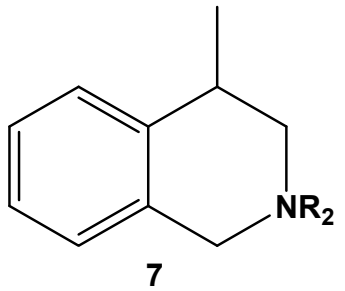

7

Table 4. Synthesis of amides 6 and 4-methyl-1,2,3,4-tetrahydroisoquinolines 7.

\begin{tabular}{cccc}
\hline $\mathbf{6}$ & $\mathbf{R}_{\mathbf{2}}$ & Yield, \% & M.p., ${ }^{\circ} \mathbf{C}$ \\
\hline $\mathbf{a}$ & $\mathrm{COCH}_{3}$ & 98 & Oil \\
b & $\mathrm{SO}_{2} \mathrm{CH}_{3}$ & 98 & Oil \\
c & $\mathrm{COPhPh}$ & 98 & $156-157$ \\
\hline $\mathbf{7}$ & $\mathbf{R}_{\mathbf{2}}$ & Yield, \% & m.p., ${ }^{\circ} \mathbf{C}$ \\
\hline a & $\mathrm{COCH}_{3}$ & 89 & Oil \\
b & $\mathrm{SO}_{2} \mathrm{CH}_{3}$ & 93 & $66-67$ \\
c & $\mathrm{COPhPh}$ & 96 & Oil \\
\hline
\end{tabular}

\section{Experimental}

\subsection{General}

Reagents and chemicals were purchased from commercial sources (Sigma-Aldrich S.A. and Riedel-de Haën) and used as received. Melting points were determined on a Boetius hot stage apparatus and are uncorrected. Spectra were recorded on a Bruker Avance DRX250 spectrometer (BAS-IOCCP, Sofia). ${ }^{1} \mathrm{H}-\mathrm{NMR}$ and ${ }^{13} \mathrm{C}-\mathrm{NMR}$ spectra were taken in $\mathrm{CDCl}_{3}$ (unless otherwise specified) at $250 \mathrm{MHz}$ and $62.5 \mathrm{MHz}$ respectively. Chemical shifts were given in part per million (ppm) relative and were referenced to TMS $(\delta=0.00 \mathrm{ppm})$ as an internal standard and coupling constants are indicated in Hz. All the NMR spectra were taken at rt (ac. $295 \mathrm{~K}$ ). Elemental analyses were performed with a vario EL III. TLC was carried out on precoated $0.2 \mathrm{~mm}$ Fluka silica gel 60 plates, using diethyl ether $n$-hexane $=1: 1$ as chromatographic system. Merck silica gel 60 (0.063-0.2 mm) was used for column chromatographic separation. Polyphosphoric acid was obtained from $85 \%$ phosphoric acid and $\mathrm{P}_{2} \mathrm{O}_{5}(1: 1 \mathrm{w} / \mathrm{w})$.

\subsection{Preparation of PPA/SiO ${ }_{2}$ Catalyst General Procedure}

PPA (4.0 g) was charged in the round-bottom flask, and $\mathrm{CHCl}_{3}(100 \mathrm{~mL})$ was added. After the mixture was stirred at $50{ }^{\circ} \mathrm{C}$ for $1 \mathrm{~h}$, followed by $\mathrm{SiO}_{2}(16.0 \mathrm{~g}, 70-230 \mathrm{mesh})$ was added to the solution, and the mixture was stirred for another $1 \mathrm{~h} . \mathrm{CHCl}_{3}$ was removed by evaporation, and the resulting solid was dried in vacuo at room temperature for $3 \mathrm{~h}$. Used $\mathrm{PPA} / \mathrm{SiO}_{2}$ was regenerated as follows: $\mathrm{PPA} / \mathrm{SiO}_{2}$ was recovered by filtration from the reaction mixture, and then it was put in the $50 \mathrm{~mL}$ round-bottom flask and dried in vacuum at $100^{\circ} \mathrm{C}$ for $2 \mathrm{~h}$. 


\subsection{Typical Procedure for the Synthesis of 2-Amino-1,1-diphenylethanes 3}

Aminoacetaldehyde dimethylacetal $(1,0,210 \mathrm{~g}, 2 \mathrm{mmol})$ was dissolved in the corresponding benzene $(22.47 \mathrm{mmol})$ in an open flask and polyphosphoric acid ( $3 \mathrm{~g})$ was added. The reaction mixture was stirred for $20 \mathrm{~min}$ at $\mathrm{rt}$. After completion of the reaction, the mixture was poured over ice. The organic layer was removed and after that the water layer the solution was neutralized with sodium hydroxide, then extracted with $\mathrm{CH}_{2} \mathrm{Cl}_{2}(3 \times 20 \mathrm{~mL})$. Combined extracts were dried $\left(\mathrm{Na}_{2} \mathrm{SO}_{4}\right)$ and concentrated. The products, after evaporation of the solvent, were purified by column chromatography on silica gel using $\mathrm{Et}_{2} \mathrm{O}$ as eluent.

\subsection{2,2-Diphenylethanamine (3a): Known Compound [53]}

2,2-Bis(3,4-dimethoxyphenyl)ethanamine (3b). ${ }^{1} \mathrm{H}-\mathrm{NMR}: 1.97$ (broad s, 2H, $\left.\mathrm{NH}_{2}\right), 3.29(\mathrm{~d}, 2 \mathrm{H}$, $J=7.6 \mathrm{~Hz}), 3.85(\mathrm{~s}, 6 \mathrm{H}), 3.87(\mathrm{~s}, 6 \mathrm{H}), 3.93(\mathrm{ddd}, 1 \mathrm{H}, J=4.0,6.0,10.0 \mathrm{~Hz}), 6.76-6.86(\mathrm{~m}, 6 \mathrm{H})$; ${ }^{13} \mathrm{C}-\mathrm{NMR}: 149.0,147.7,135.3,119.7,111.6,111.3,55.9,53.8,47.1$; Anal. calc. for $\mathrm{C}_{18} \mathrm{H}_{23} \mathrm{NO}_{4}: \mathrm{C}_{\text {, }}$ $68.12 ; \mathrm{H}, 7.30 ; \mathrm{N}, 4.41$; found $\mathrm{C}, 68.18 ; \mathrm{H}, 7.21 ; \mathrm{N}, 4.38$.

\subsection{Acylation of Amines 3: Typical Procedure for the Synthesis of Amides $\mathbf{4}$ and $\mathbf{6}$}

To solution of amine 3 ( $1 \mathrm{mmol})$ in dichloromethane $(15 \mathrm{~mL})$ equal amount of acetyl chloride, methanesulfonyl chloride or biphenyl-4-carbonyl chloride was added. After 10 min a little excess of triethylamine was added. After $30 \mathrm{~min}$ the solution was washed with diluted hydrochloric acid, saturated solution of $\mathrm{Na}_{2} \mathrm{CO}_{3}$ and water. The organic layer was dried $\left(\mathrm{Na}_{2} \mathrm{SO}_{4}\right)$, concentrated and filtered on short column with neutral $\mathrm{Al}_{2} \mathrm{O}_{3}$.

$N$-(2,2-Diphenylethyl)acetamide (4a). ${ }^{1} \mathrm{H}-\mathrm{NMR}: 1.87$ (s, 3H), 3.88 (dd, 2H, $\left.J=5.9,7.9 \mathrm{~Hz}\right), 4.17$ $(\mathrm{t}, 1 \mathrm{H}, J=8.0 \mathrm{~Hz}), 5.46(\mathrm{~s}, 1 \mathrm{H}, \mathrm{NH}), 7.22-7.25(\mathrm{~m}, 6 \mathrm{H}), 7.28-7.31(\mathrm{~m}, 4 \mathrm{H}) ;{ }^{13} \mathrm{C}-\mathrm{NMR}: 170.1,141.9$, 128.8, 128.1, 126.9, 50.6, 43.9, 23.3; Anal. calc. for $\mathrm{C}_{16} \mathrm{H}_{17} \mathrm{NO}$ : C, 80.30; H, 7.16; N, 5.85; found C, 80.26; H, 7.23; N, 5.78 .

$N$-(2,2-Diphenylethyl)methanesulfonamide (4b). ${ }^{1} \mathrm{H}-\mathrm{NMR}: 2.81$ (s, 3H), 3.76 (d, 2H, J= $\left.7.9 \mathrm{~Hz}\right), 4.20$ (t, 1H, $J=8.0 \mathrm{~Hz}), 7.20-7.28(\mathrm{~m}, 6 \mathrm{H}), 7.29-7.36$ (m, 4H); ${ }^{13} \mathrm{C}-\mathrm{NMR}: 140.9,129.0,128.0,127.3,51.4$, 47.6, 40.5; Anal. calc. for $\mathrm{C}_{15} \mathrm{H}_{17} \mathrm{NO}_{2} \mathrm{~S}$ : C, 65.43; H, 6.22; N, 5.09; S, 11.64; found C, 65.37; H, 6.29; $\mathrm{N}, 5.01 ; \mathrm{S}, 11,71$.

$N$-(2,2-Diphenylethyl)-[1,1'-biphenyl]-4-carboxamide (4c). ${ }^{1} \mathrm{H}-\mathrm{NMR} \quad\left(\mathrm{CF}_{3} \mathrm{COOD}\right): 4.36$ (d, 2H, $J=7.8 \mathrm{~Hz}), 4.48(\mathrm{dd}, 1 \mathrm{H}, J=7.0,9.4 \mathrm{~Hz}), 7.26-7.36(\mathrm{~m}, 3 \mathrm{H}), 7.35(\mathrm{~s}, 5 \mathrm{H}), 7.38-7.49(\mathrm{~m}, 5 \mathrm{H})$, 7.55-7.72 (m, 6H); ${ }^{13} \mathrm{C}-\mathrm{NMR}: 148.4,139.9,138.4,128.7,128.6,128.5,127.7,127.5,127.4,127.2$, 126.6, 121.0, 112.0, 107.5, 49.8, 46.5; Anal. calc. for $\mathrm{C}_{27} \mathrm{H}_{23} \mathrm{NO}$ : $\mathrm{C} 85.91 ; \mathrm{H}, 6.14 ; \mathrm{N}, 3.71$; found $\mathrm{C}$ 85.97; H, 6.08; N, 3.78 .

$N$-(2,2-Bis(3,4-dimethoxyphenyl)ethyl)acetamide (4d). ${ }^{1} \mathrm{H}-\mathrm{NMR}: 1.91$ (s, 3H), 3.82-3.90 (m, 2H), overlapped with $3.85(\mathrm{~s}, 6 \mathrm{H}), 3.87(\mathrm{~s}, 6 \mathrm{H}), 4.08(\mathrm{t}, 1 \mathrm{H}, J=7.9 \mathrm{~Hz}), 5.46(\mathrm{t}, 1 \mathrm{H}, J=5.7 \mathrm{~Hz}, \mathrm{NH}), 6.76$ $(\mathrm{dd}, 2 \mathrm{H}, J=1.9,5.8 \mathrm{~Hz}), 6.81(\mathrm{~d}, 2 \mathrm{H}, J=1.9 \mathrm{~Hz}), 6.83(\mathrm{~s}, 1 \mathrm{H}), 6.86(\mathrm{~s}, 1 \mathrm{H}) ;{ }^{13} \mathrm{C}-\mathrm{NMR}: 170.0,149.1$, 
147.9, 134.5, 119.7, 111.4, 111.3, 55.9, 55.8, 49.6, 44.0, 23.3; Anal. calc. for $\mathrm{C}_{20} \mathrm{H}_{25} \mathrm{NO}_{5}$ : C, 66.83; $\mathrm{H}$, $7.01 ; \mathrm{N}, 3.90$; found C, 66.75; H, 7.09; N, 3.86.

$\mathrm{N}$-(2,2-Bis(3,4-dimethoxyphenyl)ethyl)methanesulfonamide (4e). ${ }^{1} \mathrm{H}-\mathrm{NMR}: 2.84(\mathrm{~s}, 3 \mathrm{H}), 3.67(\mathrm{~d}, 2 \mathrm{H}$, $J=7.7 \mathrm{~Hz}), 3.83$ (s, 3H), 3.85 (s, 3H), 4.09 (t, 1H, $J=7.8 \mathrm{~Hz}), 4.32$ (broad s, 1H, NH), 6.74 (dd, 2H, $J=1.8,9.8 \mathrm{~Hz}), 6.80(\mathrm{~d}, 2 \mathrm{H}, J=1.9 \mathrm{~Hz}), 6.83(\mathrm{~d}, 2 \mathrm{H}, J=8.2 \mathrm{~Hz}) ;{ }^{13} \mathrm{C}-\mathrm{NMR}: 149.2,148.2,133.6$, 119.6, 111.5, 111.4, 56.0, 55.9, 50.4, 47.7, 40.4; Anal. calc. for $\mathrm{C}_{19} \mathrm{H}_{25} \mathrm{NO}_{6} \mathrm{~S}: \mathrm{C}, 57.70 ; \mathrm{H}, 6.37$; $\mathrm{N}$, 3.54 ; S, 8.11; found C, 57.78; H, 6.32; N, 3.61; S, 8.01.

$N$-(2,2-Bis(3,4-dimethoxyphenyl)ethyl)-[1,1'-biphenyl]-4-carboxamide (4f). ${ }^{1} \mathrm{H}-\mathrm{NMR}: 3.82$ (s, 6H), $3.86(\mathrm{~s}, 6 \mathrm{H}), 4.05(\mathrm{dd}, 2 \mathrm{H}, J=5.7,7.7 \mathrm{~Hz}), 4.24(\mathrm{t}, 1 \mathrm{H}, J=7.9 \mathrm{~Hz}), 6.20$ (t, 1H, $J=5.5 \mathrm{~Hz}, \mathrm{NH}), 6.82$ $(\mathrm{dd}, 6 \mathrm{H}, J=1.1,10.9 \mathrm{~Hz}), 7.36-7.47(\mathrm{~m}, 3 \mathrm{H}), 7.54-7.70(\mathrm{~m}, 6 \mathrm{H}) ;{ }^{13} \mathrm{C}-\mathrm{NMR}: 167.2,149.2,148.1$, $144.3,139.9,134.6,133.2,128.9,128.0,127.3,120.0,111.6,111.4,55.9,50.0,44.6$; Anal. calc. for $\mathrm{C}_{31} \mathrm{H}_{31} \mathrm{NO}_{5}$ : C, 74.83; H, 6.28; N, 2.81; found C, 74.75; H, 6.34; N, 2.79.

$N$-(2-Phenylpropyl)acetamide (6a). ${ }^{1} \mathrm{H}-\mathrm{NMR}: 1.20$ (d, 3H, $\left.J=7.0 \mathrm{~Hz}\right), 1.87(\mathrm{~s}, 3 \mathrm{H}), 2.86$ (ddd, 1H, $J=2.2,7.0,13.2 \mathrm{~Hz}), 3.14(\mathrm{ddd}, 1 \mathrm{H}, J=4.8,8.8,13.4 \mathrm{~Hz}), 3.56(\mathrm{ddd}, 1 \mathrm{H}, J=6.1,7.0,13.1 \mathrm{~Hz}), 5.30$ (broad s, $1 \mathrm{H}, \mathrm{NH}), 7.14(\mathrm{ddd}, 3 \mathrm{H}, J=1.8,4.3,5.5 \mathrm{~Hz}), 7.25(\mathrm{dt}, 2 \mathrm{H}, J=2.0,3.5 \mathrm{~Hz}) ;{ }^{13} \mathrm{C}-\mathrm{NMR}$ : 170.0, 144.1, 128.8, 127.2, 126.8, 46.2, 39.7, 23.3, 19.5; Anal. calc. for $\mathrm{C}_{11} \mathrm{H}_{15} \mathrm{NO}$ : C, 74.54; H, 8.53; N, 7.90; found C, 74.48; H, 8.60; N, 7.85.

$N$-(2-Phenylpropyl)methanesulfonamide (6b). ${ }^{1} \mathrm{H}-\mathrm{NMR}: 1.24$ (d, 3H, $\left.J=7.0 \mathrm{~Hz}\right), 2.69$ (s, 3H), 2.89 (dd, $1 \mathrm{H}, J=6.7,14.7 \mathrm{~Hz}), 3.11-3.19(\mathrm{~m}, 1 \mathrm{H}), 3.21-3.33(\mathrm{~m}, 1 \mathrm{H}), 4.22(\mathrm{t}, 1 \mathrm{H}, J=6.8 \mathrm{~Hz}, \mathrm{NH}), 7.13-7.21$ (m, 3H), 7.23-7.30 (m, 2H); ${ }^{13} \mathrm{C}-\mathrm{NMR}: 143.1,128.9$, 127.3, 127.2, 50.0, 40.4, 40.3, 19.0; Anal. calc. for $\mathrm{C}_{10} \mathrm{H}_{15} \mathrm{NO}_{2} \mathrm{~S}$ : C, 56.31; H, 7.09; N, 6.57; S, 15.03; found C, 56.26; H, 7.15; N, 6.49; S, 15.09.

$N$-(2,2-Bis(3,4-dimethoxyphenyl)ethyl)-[1,1'-biphenyl]-4-carboxamide (6c). ${ }^{1} \mathrm{H}-\mathrm{NMR}: 1.28$ (d, 3H, $J=7.0 \mathrm{~Hz}$ ), 2.95-3.09 (m, 1H), 3.35 (ddd, $1 \mathrm{H}, J=4.9,8.7,13.5 \mathrm{~Hz}), 3.78$ (ddd, $1 \mathrm{H}, J=6.1,6.9,13.2 \mathrm{~Hz}$ ), $6.00(\mathrm{t}, 1 \mathrm{H}, J=5.8 \mathrm{~Hz}, \mathrm{NH}), 7.15-7.20(\mathrm{~m}, 3 \mathrm{H}), 7.25-7.32(\mathrm{~m}, 3 \mathrm{H}), 7.33-7.39(\mathrm{~m}, 2 \mathrm{H}), 7.59-7.63(\mathrm{~m}, 2 \mathrm{H})$; ${ }^{13} \mathrm{C}-\mathrm{NMR}: 167.2$, 144.2, 144.1, 140.0, 133.4, 128.93, 128.85, 128.0, 127.34, 127.29, 127.2, 127.0, 46.7, 39.9, 19.3; Anal. calc. for $\mathrm{C}_{31} \mathrm{H}_{31} \mathrm{NO}_{5}$ : C, 74.83; H, 6.28; N, 2.81; found C, 74.79; H, 6.33; N, 2.78 .

3.6. Cyclization of Amides $\mathbf{4}$ and $\mathbf{6}$ in Acetic/Trifluoracetic Acid Milieu: Typical Procedure

2-Phenylethylamides $(3 \mathrm{mmol})$ and paraformaldehyde $(5 \mathrm{mmol})$ were dissolved in a mixture of $\mathrm{CH}_{3} \mathrm{COOH} / \mathrm{CF}_{3} \mathrm{COOH}=4: 1(5 \mathrm{~mL})$ at $80-100{ }^{\circ} \mathrm{C}$ for $1 \mathrm{~h}$. The solution was poured on the crushed ice and extracted with $\mathrm{CH}_{2} \mathrm{Cl}_{2}(3 \times 20 \mathrm{~mL})$. The extract was washed with $20 \%$ aq. $\mathrm{Na}_{2} \mathrm{CO}_{3}(2 \times 30 \mathrm{~mL})$ and dried $\left(\mathrm{Na}_{2} \mathrm{SO}_{4}\right)$. The solvent was distilled and the products were purified by recrystallization $\left(\mathrm{Et}_{2} \mathrm{O}\right)$.

\subsection{Cyclization of Amides $\mathbf{4}$ and $\mathbf{6}$ in $\mathrm{SiO}_{2}$-Supported Milieu: Typical Procedure}

2-Phenylethylamides ( $3 \mathrm{mmol})$ and paraformaldehyde $(5 \mathrm{mmol})$ were dissolved in a $\mathrm{C}_{2} \mathrm{H}_{4} \mathrm{Cl}_{2}(10 \mathrm{~mL})$ at $80{ }^{\circ} \mathrm{C}, 0.06 \mathrm{~g}$ of catalyst $\left(\mathrm{SiO}_{2} / \mathrm{PPA}\right)$ and $1 \mathrm{~h}$ reflux. After the completion of the reaction the reaction mixture was cooled and the catalyst was separated by simple filtration. 
1-(4-Phenyl-3,4-dihydroisoquinolin-2(1H)-yl)ethanone (5a). ${ }^{1} \mathrm{H}-\mathrm{NMR}: 1.67(\mathrm{~s}, 3 \mathrm{H}), 3.82$ (d, 2H, $J=4.4 \mathrm{~Hz}), 4.23(\mathrm{t}, 1 \mathrm{H}, J=4.2 \mathrm{~Hz}), 4.52(\mathrm{~d}, 1 \mathrm{H}, J=17.1 \mathrm{~Hz}), 5.23(\mathrm{~d}, 1 \mathrm{H}, J=17.4 \mathrm{~Hz}), 6.96-7.06$ (m, 3H), 7.22-7.29 (m, 6H); ${ }^{13} \mathrm{C}-\mathrm{NMR}: 169.9,142.7,136.0,133.6,129.5,128.7,128.5,127.1,126.6$, 51.2, 45.4, 44.3, 20.8; Anal. calc. for C17H17NO: C, 81.24; H, 6.82; N, 5.57; O, 6.37; found C, 81.28; H, 6.77; N, 5.60.

2-(Methylsulfonyl)-4-phenyl-1,2,3,4-tetrahydroisoquinoline (5b). ${ }^{1} \mathrm{H}-\mathrm{NMR}: 3.47$ (dd, 1H, $\left.J=7.4,12.5 \mathrm{~Hz}\right)$, 3.86 (ddd, 1H, $J=1.0,5.0,12.4 \mathrm{~Hz}$ ), 4.33 (t, 1H, $J=6.3 \mathrm{~Hz}$ ), 4.57 (q, 2H, $J=15.4,15.38 \mathrm{~Hz}$ ), 6.94-6.98 (m, 1H), 7.11-7.18 (m, 4H), 7.21-7.31 (m, 4H); ${ }^{13} \mathrm{C}-\mathrm{NMR}: 142.5,136.2,132.2,129.9$, 128.9, 128.7, 127.2, 127.0, 126.3, 50.9, 47.6, 45.1, 36.4; Anal. calc. for $\mathrm{C}_{16} \mathrm{H}_{17} \mathrm{NO}_{2} \mathrm{~S}: \mathrm{C}, 66.87$; $\mathrm{H}$, 5.96; N, 4.87; S, 11.16; found C, 66.91; H, 5.91; N, 4.90; S, 11.11.

Biphenyl-4-yl(4-phenyl-3,4-dihydroisoquinolin-2(1H)-yl)methanone (5c). ${ }^{1} \mathrm{H}-\mathrm{NMR}\left(\mathrm{CF}_{3} \mathrm{COOD}\right): 4.36$ $(\mathrm{d}, 2 \mathrm{H}, J=7.8 \mathrm{~Hz}), 4.48(\mathrm{dd}, 1 \mathrm{H}, J=7.0,9.4 \mathrm{~Hz}), 7.26-7.36(\mathrm{~m}, 3 \mathrm{H}), 7.35(\mathrm{~s}, 5 \mathrm{H}), 7.38-7.49(\mathrm{~m}, 5 \mathrm{H})$, 7.55-7.72 (m, 6H); ${ }^{13} \mathrm{C}-\mathrm{NMR}: 148.4,139.9,138.4,128.7,128.6,128.5,127.7,127.5,127.4,127.2$, 126.6, 121.0, 112.0, 107.5, 49.8, 46.5; Anal. calc. for $\mathrm{C}_{28} \mathrm{H}_{23} \mathrm{NO} \mathrm{C}, 86.34 ; \mathrm{H}, 5.95 ; \mathrm{N}, 3.60$; found C, 86.39; H, 5.89; N, 3.58.

1-(4-(3,4-Dimethoxyphenyl)-6,7-dimethoxy-3,4-dihydroisoquinolin-2(1H)-yl)ethanone (5d). ${ }^{1} \mathrm{H}-\mathrm{NMR}$ $\left(\mathrm{CDCl}_{3}\right): 1.66(\mathrm{~s}, 3 \mathrm{H}), 3.74(\mathrm{~s}, 3 \mathrm{H}), 3.81(\mathrm{~s}, 3 \mathrm{H}), 3.87(\mathrm{~s}, 3 \mathrm{H}), 3.90(\mathrm{~s}, 3 \mathrm{H}), 4.90(\mathrm{t}, 1 \mathrm{H}, J=0.96 \mathrm{~Hz})$, $4.93(\mathrm{~d}, 2 \mathrm{H}, J=1.09 \mathrm{~Hz}), 5.34-5.35(\mathrm{~m}, 2 \mathrm{H}), 6.47(\mathrm{~s}, 1 \mathrm{H}), 6.53(\mathrm{~s}, 1 \mathrm{H}), 6.68-6.69(\mathrm{~m}, 2 \mathrm{H}), 6.82(\mathrm{~s}$, $1 \mathrm{H}) ;{ }^{13} \mathrm{C}-\mathrm{NMR}\left(\mathrm{CDCl}_{3}\right): 169.9,149.0,148.3,148.1,148.0,147.9,135.5,120.7,111.8,111.4,111.2$, 109.2, 108.6, 56.2, 56.0, 55.93, 55.89, 51.5, 44.6, 43.9, 20.9. Anal. calc. for $\mathrm{C}_{21} \mathrm{H}_{25} \mathrm{NO}_{5}$ : C, 67.91; $\mathrm{H}$, $6.78 ; \mathrm{N}, 3.77$; found $\mathrm{C}, 67.96 ; \mathrm{H}, 6.74 ; \mathrm{N}, 3.82$.

4-(3,4-Dimethoxyphenyl)-6,7-dimethoxy-2-(methylsulfonyl)-1,2,3,4-tetrahydroisoquinoline (5e). ${ }^{1} \mathrm{H}-\mathrm{NMR}$ $\left(\mathrm{CDCl}_{3}\right): 2.72(\mathrm{~s}, 3 \mathrm{H}), 3.41(\mathrm{dd}, 1 \mathrm{H}, J=7.42,12.37 \mathrm{~Hz}), 3.72(\mathrm{~s}, 3 \mathrm{H}), 3.81-3.86(\mathrm{~m}, 1 \mathrm{H})$, overlapped with $3.84(\mathrm{~s}, 3 \mathrm{H}), 3.89(\mathrm{~s}, 3 \mathrm{H}), 3.90(\mathrm{~s}, 3 \mathrm{H}), 4.21(\mathrm{dd}, 1 \mathrm{H}, J=5.58,6.24 \mathrm{~Hz}), 4.51(\mathrm{q}, 2 \mathrm{H}, J=6.25$, $15.0 \mathrm{~Hz}), 6.46(\mathrm{~s}, 1 \mathrm{H}), 6.64(\mathrm{~s}, 1 \mathrm{H}), 6.67-6.71(\mathrm{~m}, 2 \mathrm{H}), 6.81-6.85(\mathrm{~m}, 1 \mathrm{H}) ;{ }^{13} \mathrm{C}-\mathrm{NMR}\left(\mathrm{CDCl}_{3}\right): 149.0$, 148.2, 148.1, 135.0, 128.2, 124.1, 121.0, 112.1, 111.9, 111.2, 108.5, 56.0, 55.9, 51.1, 47.3, 44.3, 36.3. Anal. calc. for $\mathrm{C}_{20} \mathrm{H}_{25} \mathrm{NO}_{6} \mathrm{~S}$ : C, 58.95; H, 6.18; N, 3.44; S, 7.87; found C, 58.99; H, 6.14; N, 3.45; S, 7.81.

Biphenyl-4-yl[4-(3,4-dimethoxyphenyl)-6,7-dimethoxy-3,4-dihydroisoquinolin-2(1H)-yl]methanone (5f). ${ }^{1} \mathrm{H}-\mathrm{NMR}: 3.73$ (s, 3H), 3.76 (s, 3H), $3.91(\mathrm{~s}, 3 \mathrm{H}), 3.94$ (s, 3H), 4.09 (s, 1H), 4.65 (d, 1H, J=17.0 Hz), $5.34(\mathrm{~d}, 1 \mathrm{H}, J=16.6 \mathrm{~Hz}), 6.51(\mathrm{~s}, 2 \mathrm{H}), 6.81(\mathrm{~d}, 2 \mathrm{H}, J=7.7 \mathrm{~Hz}), 6.92(\mathrm{~d}, 2 \mathrm{H}, J=6.8 \mathrm{~Hz}), 7.36-7.50$ (m, 6H), 7.57-7.65 (m, 4H); ${ }^{13} \mathrm{C}-\mathrm{NMR}: 171.3,149.2,148.5,148.14,148.07,142.2,140.2,135.2$, $134.5,128.9,127.5,127.4,127.1,126.8,125.3,112.0,111.7,111.2,108.8,56.03,55.99,55.94,55.87$, 52.2, 51.8, 44.6. Anal. calc. for $\mathrm{C}_{32} \mathrm{H}_{31} \mathrm{NO}_{5}$ : C, 75.42; H, 6.13; N, 2.75; found C, 75.50; H, 6.10; N, 2.81 .

\section{Conclusions}

In conclusion, we have developed a highly efficient $\mathrm{SiO}_{2}$-PPA catalyzed method for the construction of 4-aryl- or 4-methyl-1,2,3,4-tetrahydroisoquinoline ring systems, as analogues of biologically-active compounds. The catalyst is completely recoverable and the efficiency of the 
catalyst remains unaltered even after three to four cycles. It is also noticed that the cyclisation using PPA- $-\mathrm{SiO}_{2}$ proceeds rapidly and is superior to the reported procedures with respect to yield and amount of the catalyst employed.

\section{Acknowledgments}

We acknowledge financial support from the National Fund for scientific research DO-02-131 and DO-02-195.

\section{References}

1. Brossi, A.; Grethe, G.; Tietel, S.; Wildman, W.C.; Bailey, D.T. Cherylline, a 4-phenyl-1,2,3,4tetrahydroisoquinoline alkaloid. J. Org. Chem. 1970, 35, 1100-1104.

2. Jacob, J.N.; Nichols, D.E.; Kohli, J.D.; Glock, D. Dopamine agonist properties of N-alkyl-4-(3,4dihydroxyphenyl)-1,2,3,4-tetrahydroisoquinolines. J. Med. Chem. 1981, 24, 1013-1015.

3. Zara-Kaczian, E.; Gyorgy, L.; Deak, G.; Seregi, A.; Doda, M. Synthesis and pharmacological evaluation of some new tetrahydroisoquinoline derivatives inhibiting dopamine uptake and/or possessing a dopaminomimetic property. J. Med. Chem. 1986, 29, 1189-1195.

4. Maryanoff, B.E.; Vaught, J.L.; Shank, R.P.; McComsey, D.F.; Costanzo, M.J.; Nortey, S.O. Pyrroloisoquinoline antidepressants. 3. A focus on serotonin. J. Med. Chem. 1990, 33, 2793-2797.

5. Kihara, M.; Ikeuchi, M.; Yamauchi, A.; Nukatsuka, M.; Matsumoto, H.; Toko, T. Synthesis and biological evaluation of 7-hydroxy-3,4-diphenyl-1,2-dihydroisoquinolines as new 4-hydroxytamoxifen analogues. Chem. Pharm. Bull. 1997, 45, 939-943.

6. Venkov, A.P.; Vodenicharov, D.; A New Synthesis of 1,2,3,4-Tetrahydro-2-methyl-4phenylisoquinolines. Synthesis 1990, 253-255.

7. Kihara, M.; Kashimoto, M.; Kobayashi, Y.; Kobayashi, S. A new intramolecular barbier reaction of N-(2-iodobenzyl)phenacylamines: A convenient synthesis of 1,2,3,4-tetrahydroisoquinomn-4-ols. Tetrahedron Lett. 1990, 31, 5347-5348.

8. Venkov, A.P.; Vodenicharov, D.M.; Ivanov, I.I. 4-Aryl-1,4-dihydro-3(2H)-isoquinolinones are obtained by oxidative cyclization of $N$-benzyl- $N$-alkylarylacetamides with lead tetra-acetate in acetic acid/trifluoroacetic acid. Synthesis 1991, 476-478.

9. Kihara, M.; Kashimoto, M.; Kobayashi, Y.; Nagao, Y. A new synthesis of 7,12-Dihydro-12phenyl-5H-6,12-methanodibenz[ $c$, $]$ azocines via $N$-Benzyl-1,2,3,4-tetrahydro-4-phenylisoquinolin-4ols. Heterocycles 1992, 34, 747-756.

10. Coskun, N.; Sümengen, D. A new synthesis of N-Substituted-1,2,3,4-tetrahydro-4phenylisoquinolin-3-ones. Synth. Commun. 1993, 23, 1393-1402.

11. Miller, R.B.; Svoboda, J.J. An efficient synthesis of 4-aryl-1,2,3,4-tetrahydroisoquinolines. Synth. Commun. 1994, 24, 1187-1194.

12. Meda, N.; Selvakumar, N.; Kraus, G.A. An efficient synthesis of 4-aryl kainic acid analogs. Tetrahedron 1999, 55, 943-954.

13. Kuster, G.J.; Kalmoua, F.; Scheeren, H.W.; de Gelder, R. A simple entry towards novel bi- and tricyclic $N$-oxy- $\beta$-lactams by high pressure promoted tandem $[4+2] /[3+2]$ cycloadditions of enol ethers and $\beta$-nitrostyrene. Chem. Commun. 1999, 855-856. 
14. Nolellino, L.; D'Ischia, M.; Prota, G. Expedient synthesis of 5,6-dihydroxyindole and derivatives via an improved $\mathrm{Zn}(\mathrm{II})$-assisted 2, $\beta$-dinitrostyrene approach. Synthesis 1999, 793-796.

15. Kihara, M.; Andoh, J.I.; Yoshida, C. New reduction reaction of benzylic alcohols with acid and proof of the intermolecular hydride shift mechanism. Heterocycles 2000, 53, 359-372.

16. Labrana, J.; Machocho, A.K.; Kricsfalusy, V.; Brun, R.; Codina, C.; Viladomat, F.; Bastida, J. Alkaloids from Narcissus angustifolius subsp. transcarpathicus (Amaryllidaceae). Phytochemistry 2002, 60, 847-856.

17. Elgorashi, E.E.; Stafford, G.J.; Jäger, A.K.; van Staden, J. Inhibition of [3H] citalopram binding to the rat brain serotonin transporter by amaryllidaceae alkaloids. Planta Med. 2006, 72, 470-473.

18. Dong, H.; Sheng, J.Z.; Lee, C.M.; Wong, T.M. Calcium antagonistic and antiarrhythmic actions of CPU-23, a substituted tetrahydroisoquinoline. Br. J. Pharmacol. 1993, 109, 113-119.

19. Bernan, V.S.; Montenegro, D.A.; Korshalla, J.D.; Maiese, W.M.; Steinberg, D.A.; Greenstein, M. Bioxalomycins, new antibiotics produced by the marine Streptomyces sp. LL-31F508: Toxonomy and fermentation. J. Antibiot. 1994, 47, 1417-1424.

20. Ratsimamanga-Urverg, S.; Rasoanaivo, P.; Rafatro, H.; Robijaona, B.; Rakoto-Ratsimamanga, A. In vitro antiplasmodial activity and chloroquine-potentiating action of three new isoquinoline alkaloid dimers isolated from Hernandia voyronii Jumelle. Ann. Trop. Med. Parasitol. 1994, 88, 271-277.

21. Riggs, R.M.; Nichols, D.E.; Foreman, M.M.; Truex, L.L. Effect of $\beta$-alkyl substitution on D-1 dopamine agonist activity: Absolute configuration of $\beta$-methyldopamine. J. Med. Chem. 1987, 30, $1887-1897$.

22. Chesworth, R.; Zawistoski, M.P.; Lefker, B.A.; Cameron, K.O.; Day, R.F.; Mangano, F.M.; Rosati, R.L.; Colella, S.; Petersen, D.N.; Brault, A.; et al. Tetrahydroisoquinolines as subtype selective estrogen agonists/antagonists. Bioorg. Med. Chem. Lett. 2004, 14, 2729-2733.

23. Keith, J.M.; Barbier, A.J.; Wilson, S.J.; Miller, K.; Boggs, J.D.; Fraser, I.C.; Mazur, C.; Lovenberg, T.W.; Carruthers, N.I. Dual serotonin transporter inhibitor/histamine H3 antagonists: Development of rigidified H3 pharmacophores. Bioorg. Med. Chem. Lett. 2007, 17, 5325-5329.

24. Brossi, A.; Teitel, S. Total synthesis of racemic cherylline. Tetrahedron Lett. 1970, 11, 417-419.

25. Ruchirawat, S.; Tontoolarug, S.; Sahakitpichan, P. Synthesis of 4-aryltetrahydroiso-quinolines: Application to the synthesis of cherylline. Heterocycles 2001, 55, 635-640.

26. Takano, S.; Akiyama, M.; Ogasawara, K. Total synthesis of ( \pm )- and (+)-latifine. J. Chem. Soc. Perkin Trans. 1 1985, 2447-2453.

27. Couture, A.; Deniau, E.; Lebrun, S.; Grandclaudon, P. Total syntheses of (+/-)-cherylline and (+/-)-latifine. J. Chem. Soc. Perkin Trans. 1 1999, 789-794.

28. Cuevas, J.-C.; Snieckus, V. $\alpha^{\prime}$-Silylated tertiary benzamides as dual ortho- and $\alpha^{\prime}$-carbanion synthons. Carbodesilylative routes to isoquinoline and dibenzoquinolizidine derivatives. Tetrahedron Lett. 1989, 30, 5837-5840.

29. Philippe, N.; Denivet, F.; Vasse, J.-L.; Sopkova-de Olivera Santos, J.; Levacher, V.; Dupas, G. Highly stereoselective Friedel-Crafts type cyclization. Facile access to enantiopure 1,4-dihydro-4phenyl isoquinolinones. Tetrahedron 2003, 59, 8049-8056.

30. Hara, H.; Shirai, R.; Hoshino, O.; Umezawa, B. A Facile Synthesis of 4-aryl-1,2,3,4tetrahydroisoquinolines: A total synthesis of $( \pm)$-cherylline. Heterocycles 1983, 20, 1945-1950. 
31. Toda, J.; Sonobe, A.; Ichikava, T.; Saitoh, T.; Horiguchi, Y.; Sano, T. Synthesis of 4-aryl-2methyl-1,2,3,4-tetrahydroisoquinolines via Pummerer-type cyclization of $N$-(arylmethyl)- $N$ methyl-2-aryl-2-(phenylsulfinyl) acetamides. Arcivoc 2000, 1, 165-180.

32. Hara, H.; Shirai, R.; Hoshino, O.; Umezawa, B. Studies on tetrahydroisoquinolines. XXV. A synthesis of 4-aryl-1,2,3,4-tetrahydroisoquinolines; Total synthesis of ( \pm )-cherylline. Chem. Pharm. Bull 1985, 33, 3107-3112.

33. Couture, A.; Deniau, E.; Grandclaudon, P.; Lebrun, S. Asymmetric synthesis of (+)- and (-)-latifine. Asymmetry 2003, 14, 1309-1316.

34. Seijas, J.A.; Vázquer-Tato, M.P.; Martínez, M.M.; Pizzolatti, M.G. Oxazoline as a useful tool in organic synthesis: Preparation of 4-aryl-1,2,3,4-tetrahydroisoquinoline alkaloid skeleton. Tetrahedron Lett. 2005, 46, 5827-5830.

35. Irie, H.; Shiina, A.; Fushimi, T.; Katakawa, J.; Fujii, N.; Yajima, H. New synthesis of isoquinoline alkaloids, thalifoline, corypalline, and cherylline. Chem. Lett. 1980, 875-878.

36. Katakawa, J.; Yoshimatsu, H.; Yoshida, M.; Zhang, Y.; Irie, H.; Yajima, H. Synthesis of Amaryllidaceae Alkaloids, ( \pm )-Cherylline and $( \pm)$-Latifine. Chem. Pharm. Bull. 1988, 36, 3928-3932.

37. Kessar, V.; Singh, P.; Chawla, R.; Kumar, P. Cyclization of ortho-halogenated N-acylbenzylamines: A formal synthesis of $( \pm)$-cherylline. Chem. Soc. Chem. Commun. 1981, 1074-1075.

38. Schwartz, M.A.; Scott, S.W. Biogenetically patterned synthesis of (+-)-cherylline. J. Org. Chem. 1971, 36, 1827-1829.

39. Kametani, T.; Takahashi, K.; Van Loc, C. Studies on the syntheses of heterocyclic compoundsDXCI: Total synthesis of $( \pm)$-cherylline and corgoine through quinonoid intermediates. Tetrahedron 1975, 31, 235-238.

40. Honda, T.; Namiki, H.; Saton, F. Palladium-catalyzed intramolecular $\delta$-lactam formation of aryl halides and amide-enolates: Syntheses of cherylline and latifine. Org. Lett. 2001, 3, 631-633.

41. Freter, K.; Dubois, E.; Thomas, A. A new tetrahydroisoquinoline synthesis. J. Heterocycl. Chem. 1970, 7, 159-169.

42. Lebrun, S.; Couture, A.; Deniau, E.; Grandclaudon, P. A new synthesis of (+)- and (-)-cherylline. Org. Biomol. Chem. 2003, 1, 1701-1706.

43. Bonacorso, H.G.; Andrighetto, R.; Zanatta, N.; Martins, M.A.P. The unexpected cyclization routes of $N, N^{\prime}$-bis(oxotrifluoroalkenyl)-1,3-phenylenediamines in polyphosphoric acid medium. Tetraheron Lett. 2010, 51, 3752-3755.

44. Ivanov, I.; Nikolova, S.; Statkova-Abeghe, S. Efficient one-pot Friedel-Crafts acylation of benzene and its derivatives with unprotected aminocarboxylic acids in polyphosphoric acid. Synth. Commun. 2006, 36, 1405-1411.

45. Ivanov, I.; Nikolova, S.; Statkova-Abeghe, S. A simple method for the synthesis of 1-substituted beta-carboline derivatives from tryptamine and carboxylic acids in polyphosphoric acid. Heterocycles 2005, 65, 2483-2492.

46. Aoyama, T.; Takido, T.; Kodomari, M. Silica gel-supported polyphosphoric acid $\left(\mathrm{PPA} / \mathrm{SiO}_{2}\right)$ as an efficient and reusable catalyst for conversion of carbonyl compounds into oxathioacetals and dithioacetals. Synlett 2004, 13, 2307-2310. 
47. Itoh, K.-I.; Aoyama, T.; Satoh, H.; Fujii, Y.; Sakamaki, H.; Takido, T.; Kodomari, M. Application of silica gel-supported polyphosphoric acid $\left(\mathrm{PPA} / \mathrm{SiO}_{2}\right)$ as a reusable solid acid catalyst to the synthesis of 3-benzoylisoxazoles and isoxazolines. Tetrahedron Lett. 2011, 52, 6892-6895.

48. Zhang, Q.; Zhang, S.; Deng, Y. Recent advances in ionic liquid catalysis. Green. Chem. 2011, 13, 2619-2637.

49. Ansari, M.I.; Hussain, M.K.; Yadav, N.; Gupta, P.K.; Hajela, K. Silica supported perchloric acid catalysed rapid N-formylation under solvent-free conditions, Tetrahedron Lett. 2012, 53, 2063-2065.

50. Verma, R.S. Solvent-free organic syntheses using supported reagents and microwave irradiation. Green. Chem. 1999, 1, 43-55.

51. Corma, A.; Garcia, H. Silica-bound homogenous catalysts as recoverable and reusable catalysts in organic synthesis. Adv. Synth. Catal. 2006, 348, 1391-1412.

52. Kantevari, S.; Bantu, R.; Nagarapu, L. $\mathrm{HClO}_{4}-\mathrm{SiO}_{2}$ and $\mathrm{PPA}-\mathrm{SiO}_{2}$ catalyzed efficient one-pot Knoevenagel condensation, Michael addition and cyclo-dehydration of dimedone and aldehydes in acetonitrile, aqueous and solvent free conditions: Scope and limitations. J. Mol. Catal. A-Chem. 2007, 269, 53-57.

53. Klumpp, D.A.; Sanchez, G.V., Jr.; Aguirre, S.L.; Zhang, Y.; de Leon, S. Chemistry of dicationic electrophiles: Superacid-catalyzed reactions of amino acetals. J. Org. Chem. 2002, 67, 5028-5031.

54. Khojastehnezhad, A.; Moeinpour, F.; Davoodnia, A. PPA-SiO ${ }_{2}$ catalyzed efficient synthesis of polyhydroquinoline derivatives through Hantzsch multicomponent condensation under solvent-free conditions. Chin. Chem. Lett. 2011, 22, 807-810.

55. Vittorio, F.; Santagati, N.A.; Duro, R.; Duro, F.; Caruso, A.; Amico Roxas, M.; Trombadore, S. Alkyl derivatives of isoquinoline. III. Synthesis and pharmacologic activity of dialkylaminoalkyl amides of 1-chloro- and 1-methoxy-3-carboxy-4-methylisoquinoline and of 3-carboxy-2,4dimethyl-1,2-dihydro-1-oxoisoquinoline. Farmaco Sci. 1984, 39, 229-245.

Sample Availability: Samples of the compounds are available from the authors.

(C) 2013 by the authors; licensee MDPI, Basel, Switzerland. This article is an open access article distributed under the terms and conditions of the Creative Commons Attribution license (http://creativecommons.org/licenses/by/3.0/). 